PRINT ISSN 1119-8362

Electronic ISSN 1119-8362
Full-text Available Online at https://www.ajol.info/index.php/jasem http://ww.bioline.org.br/ja
J. Appl. Sci. Environ. Manage.

Vol. 24 (2) 381-391 February 2020

\title{
Geochemical characterization of FE-1 well, Onshore Western Niger Delta Basin, Nigeria
}

\author{
${ }^{1}$ AMIEWALAN, FO; ${ }^{2}$ LUCAS, FA \\ Department of Geology, University of Benin, Benin City, Nigeria. \\ *Corresponding Author Email: amiewalanflorence@gmail.com; drfalucas@gmail.com
}

\begin{abstract}
Major oxides and trace elements analysis was carried out on ditch cutting samples of FE-1 well, Onshore, Niger Delta Basin, to define their oxygen condition, paleo-climatic conditions, provenance, tectonic setting, sandstone classification and maturity of the sediments. Sedimentological analysis revealed five main lithofacies comprising of sandstone, shaly sand, mudstone, sandy clay and sandy shale. Geochemical results indicates that the average value of silica $(87.1 \mathrm{wt} . \%)$ is far higher than the average values of the rest oxides which shows the duration and intensity of weathering and destruction of other minerals during transportation. Bivariate plot of $\mathrm{La} / \mathrm{Sc}$ versus $\mathrm{Th} / \mathrm{Co}, \mathrm{Zr}$ versus $\mathrm{TiO}_{2}$, and trace elemental ratios of $\mathrm{Th} / \mathrm{Sc}, \mathrm{Th} / \mathrm{Co}, \mathrm{Th} / \mathrm{Cr}, \mathrm{Cr} / \mathrm{Th}, \mathrm{La} / \mathrm{Sc}$ establishes that the source of the sediment are from felsic rock. Different chemical classification schemes characterized the sediments as quartzarenite, lithic arenites and Fe-rich sand. The plot of $\mathrm{Log}\left(\mathrm{K}_{2} \mathrm{O} / \mathrm{Na}_{2} \mathrm{O}\right)$ versus $\log \left(\mathrm{SiO}_{2} / \mathrm{Al}_{2} \mathrm{O}_{3}\right)$ and $\mathrm{K}_{2} \mathrm{O} / \mathrm{Na}_{2} \mathrm{O}$ versus $\mathrm{SiO}_{2}$ all plotted mainly in the passive margin zone. The low average value of uranium content, trace elemental ratios of $\mathrm{U} / \mathrm{Th}, \mathrm{Ni} / \mathrm{Co}, \mathrm{Cu} / \mathrm{Zn}, \mathrm{V} / \mathrm{Cr}$ and binary plot of $\mathrm{Ni} / \mathrm{Co}$ versus $\mathrm{V} / \mathrm{Cr}$ indicated an oxic environment of deposition. However, the binary plot of $\mathrm{Ni} / \mathrm{Co}$ versus $\mathrm{V} /(\mathrm{V}+\mathrm{Ni})$ indicates oxic to anoxic condition during deposition of the mixed marine and terrigenous source input sediment. Binary plot of $\mathrm{SiO}_{2}$ versus $\left(\mathrm{Al}_{2} \mathrm{O}_{3}+\mathrm{Na}_{2} \mathrm{O}+\mathrm{K}_{2} \mathrm{O}\right)$ designates deposition mainly in humid/semi-humid climatic condition. The samples studied have a much higher $\mathrm{SiO}_{2} / \mathrm{Al}_{2} \mathrm{O}_{3}$ ratio with low $\mathrm{Fe}_{2} \mathrm{O}_{3} / \mathrm{K}_{2} \mathrm{O}$ ratio, thus, they are mineralogically mature. The $\mathrm{Al}_{2} \mathrm{O}_{3} /\left(\mathrm{CaO}+\mathrm{MgO}+\mathrm{Na}_{2} \mathrm{O}+\mathrm{K}_{2} \mathrm{O}\right)$ ratio inferred that there are stable mobile oxides in the samples.
\end{abstract}

DOI: https://dx.doi.org/10.4314/jasem.v24i2.26

Copyright: Copyright $(\mathcal{C} 2020$ Amiewalan and Lucas. This is an open access article distributed under the Creative Commons Attribution License (CCL), which permits unrestricted use, distribution, and reproduction in any medium, provided the original work is properly cited.

Dates: Received: 16 November 2019; Revised: 11 January 2020; Accepted: 22 February 2020

Keywords: Major oxides, lithic arenites, provenance, tectonic setting.

The Niger Delta is an essential sedimentary basin in Nigeria known to have major delta systems and utmost productive petroleum provinces worldwide. This makes Nigeria the greatest prolific oil producer in SubSaharan Africa, position as the third main producer of crude oil in Africa and the tenth leading in the world. According to Adenugba and Dipo, (2013), the Nigeria's economy is mainly reliant on its oil sector which make up about $95 \%$ of Nigeria's foreign exchange earnings and about $80 \%$ of its financial incomes. Due to the discovery of oil in commercial quantity in Oloibiri-1 well in 1956, rigorous exploration and exploitation of hydrocarbon in the basin has been ongoing since the early 1960s. Nwajide and Reijers, (1996). Based on the petroliferous nature of the Basin, there has been constant and steady geologic research for both academic and commercial drives for many years. Adebayo, (2011). Major oxides and trace element abundances in sedimentary rocks have added significantly to our understanding of paleo oxygen and paleo-climatic conditions, provenance, tectonic setting, sandstone classification and maturity of sediments. E.g. Obasi et al., (2019); Nath et al., (1997); Jones and Manning, (1994). The aim of this study is to infer the paleo-environment and paleoclimatic conditions, provenance, tectonic setting, sandstone classification and maturity of sediments during the deposition in the considered section of the Niger Delta basin. To attain the objective, a multidisciplinary method of relating sedimentological features, and inorganic geochemical data with their elemental ratio were engaged.

The geologic setting of the basin: The present-day Niger Delta Complex is situated on the continental margin of the Gulf of Guinea in the southern part of Nigeria. It lies between longitudes $4^{\circ} \mathrm{E}$ and $8.8^{\circ} \mathrm{E}$ and latitudes $3{ }^{\circ} \mathrm{N}$ and $6^{\circ} \mathrm{N}$. Reijers et al., (1997). The onshore portion of the basin is delineated by the geology of southern Nigeria and southwestern Cameroon. It is bounded in the north by outcrops of the Anambra Basin and the Abakaliki Anticlinorium, and delimited in the west by the Benin Flank, a northeast-southwest trending hinge line south of the West African basement massif. The Calabar Flank, a hinge line bordering the Oban massif, defines the northeastern boundary. The offshore boundary of the basin is defined by the Cameroon volcanic line to the

*Corresponding Author Email: amiewalanflorence@gmail.com 
east and the eastern boundary of the Dahomey Basin (the easternmost West African transform-fault passive margin) to the west. The evolution of the delta is controlled by pre and synsedimentary tectonics as described by Knox and Omatsola, (1987). It is a large arcuate delta covering an area of about $300,000 \mathrm{~km}^{2}$
Kulke, (1995), with a sediment volume of 500,000 $\mathrm{km}^{3}$, Hospers, (1965) and a sedimentary thickness of over $10 \mathrm{~km}$ in the basin Depo center. Kaplan et al., (1994). Well FE-1 is located in the Greater Ughelli Depobelt of the Niger delta. (Fig. 1).

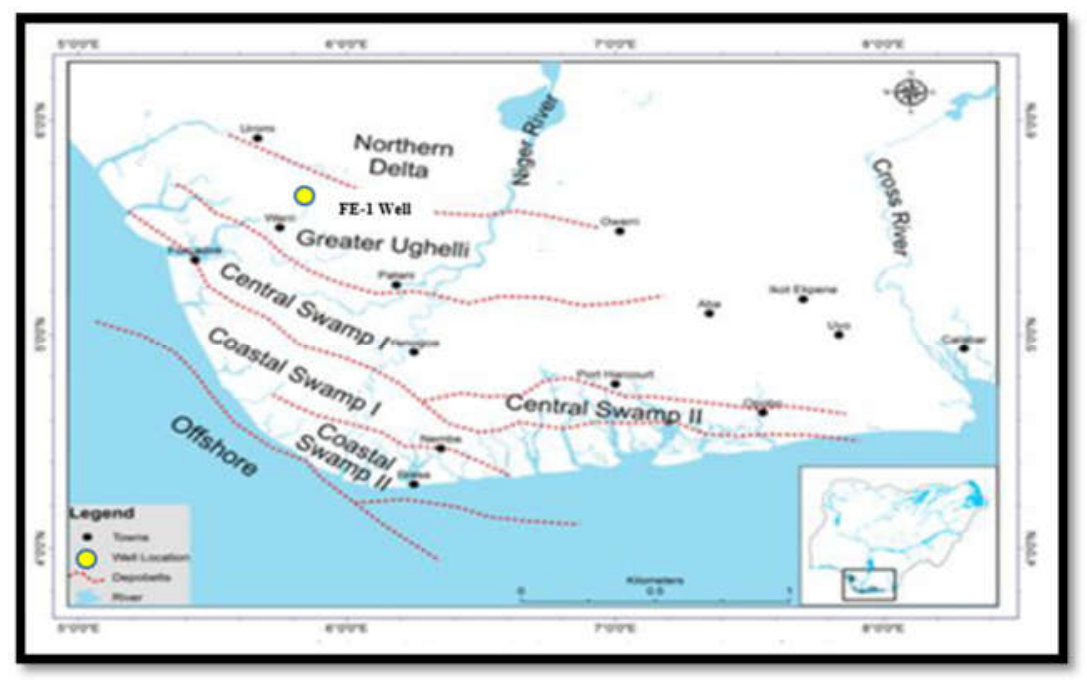

Fig. 1: A map of Niger delta Depobelts showing location of "FE-1" well.

Tectonic setting of the Niger Delta Basin: Given to Whiteman (1982), the Niger Delta Basin clastic segment was formed along aulacogen that formerly advanced in the course of breakdown of the South American and African plates during the Tithonian Kimmeridgian (latest Jurassic) to Early Cretaceous. Cretaceous fracture zones conveyed as trenches and ridges in the deep Atlantic define the tectonic framework of the continental margin along the western coast of Africa. The fracture zone ridges divided the margin into individual Basins which form the boundary faults of the Cretaceous Benue Trough that cuts far into the West African Shield. The Benue Trough has experienced a succession of depositional phases, local folding, uplifts and downwarping from the time of its opening for the period of Early Cretaceous (Aptian) to Paleocene. Subsequently, the deltaic sedimentation come to be developed in the southern part of the Benue Trough that assembled in the establishment of the current Niger Delta Basin. Short and Stauble, (1967). Reijers et al., (1997), considered the Niger Delta to be the youngest among the chain of sub-basins in the Benue Trough. On the other hand, Avbovbo and Ayoola, (1981), defined the Niger Delta Basin as that Cenozoic gross offlap clastic succession built out on top of the Anambra Basin. The Anambra Basin underlies the Niger Delta basin and the two are demarcated by an unconformity.
The stratigraphic build up have been measured by the interaction amongst the degrees of sediment supply and subsidence. Deep wells in the basin record three lithostratigraphic succession consisting of substantial and repetitions of marine shale (Akata Formation) and grade upward into interbedded sandstone and shale facies (Agbada Formation) which form the characteristic paralic facies portion of the Delta. The uppermost portion (Benin Formation) of the sequence is a considerable terrestrial sandstone segment. The lithologies of the Cenozoic Niger Delta Basin is generally categorized as continental, transitional and marine identified regularly on the basis of sand-shale ratios. The Clay members are erosional surfaces that are locally in the form of entrenchments, filled with clay deposits recognized only in the subsurface. Afam Clay, Opuama, Buguma, Qua-Iboe members. Figure

\section{MATERIAL AND METHOD}

Six hundred and seventy-eight ditch cutting samples from depths of $2700 \mathrm{ft}$. - 10,185 ft. intervals were subjected to sedimentological analyses while selected twelve ditch cuttings samples were analyzed geochemically with fused bead X-Ray Fluorescence (XRF) spectrometry for the major oxides and fused bead laser ablation inductively couple plasma mass spectroscopy (LA-ICP-MS) for the trace elements at 
Bureau Veritas Minerals laboratory, Vancouver, Canada.

Sedimentological analysis: The processed samples were studied and described by both visual inspection and the use of a reflected light binocular microscope. Physical characteristics such as the lithology, color, texture (grain size, shape and sorting), fossils and accessory constituents (mica flakes, carbonaceous detritus, ferruginous material and calcite) were determined. Dilute Hydrochloric acid was used to test for the presence of carbonate in sample.

$X R F$ and LA-ICPMS analyses: Loss on ignition (LOI) results were determined from the total weight of sample by ignition at $1000{ }^{\circ} \dot{\mathrm{C}}$ for 1 hour using a robotic TGA system. Pulverized ditch cutting samples were casted using 12.22 flux with $4 \%$ Lithium nitrate which were added to sample to form glass bead. Major oxides were determined by X-Ray Fluorescence Spectrometry on oven dry of $\left(105^{\circ} \mathrm{C}\right)$. The same set of samples was further analyzed for trace elements using LA-ICP-MS instrumental analyzes. The laser was used to vaporize the surface of the fused sample, while the vapor and any particles were then transported by the carrier gas flow to the plasma of the ICP-MS unit. The rays through the quadrupole were detected and the data passes through quality assurance and check. LA-ICPMS capitalizes a high productivity robotic fusion technology which provide a fully extracted quantitative analysis for all elements. Detection limits are comparable to industry leading mixed acid trace element ICP-MS packages. Ten major elements, $\left(\mathrm{SiO}_{2}, \mathrm{TiO}_{2}, \mathrm{Al}_{2} \mathrm{O}_{3}, \mathrm{Fe}_{2} \mathrm{O}_{3}, \mathrm{MgO}, \mathrm{MnO}, \mathrm{CaO}, \mathrm{Na}_{2} \mathrm{O}\right.$, $\mathrm{K}_{2} \mathrm{O}$ and $\mathrm{P}_{2} \mathrm{O}_{5}$ ) reported as oxide percent by weight and 33 trace elements (Ag, As, Ba, Be, Bi, Cd, Ce, Co, Cr, $\mathrm{Cs}, \mathrm{Cu}, \mathrm{Ga}, \mathrm{Hf}$, In, La, Mo, Nb, Ni, Pb, Sb, Sc, Sn, Sr, Ta, Te, Th, Tl, U, V, W, Y, Zn and Zr) reported in ppm. The post-Archean Australian shale (PAAS) and Upper Continental Crust (UCC) values were used for evaluation.

\section{RESULTS AND DISCUSSION}

Sedimentological Result: Lithologically, the sequence is categorized by the alternation of sandstone, mudstone, shaly sand, sandy shale and sandy clay facies. The shales are light grey, grey, light brown and brown, fissile and moderately hard. The sands are milky white, light grey, grey, light brown and brown, fine, medium and occasionally coarse - pebble grained, subangular, subrounded and rounded, mostly subangular. Poorly, moderately to well sorted. Index minerals and accessories are dominated by mica flakes and carbonaceous detritus with rare ferruginous material. Some of the sediments within this interval are calcareous while some are not calcareous. Table 1.

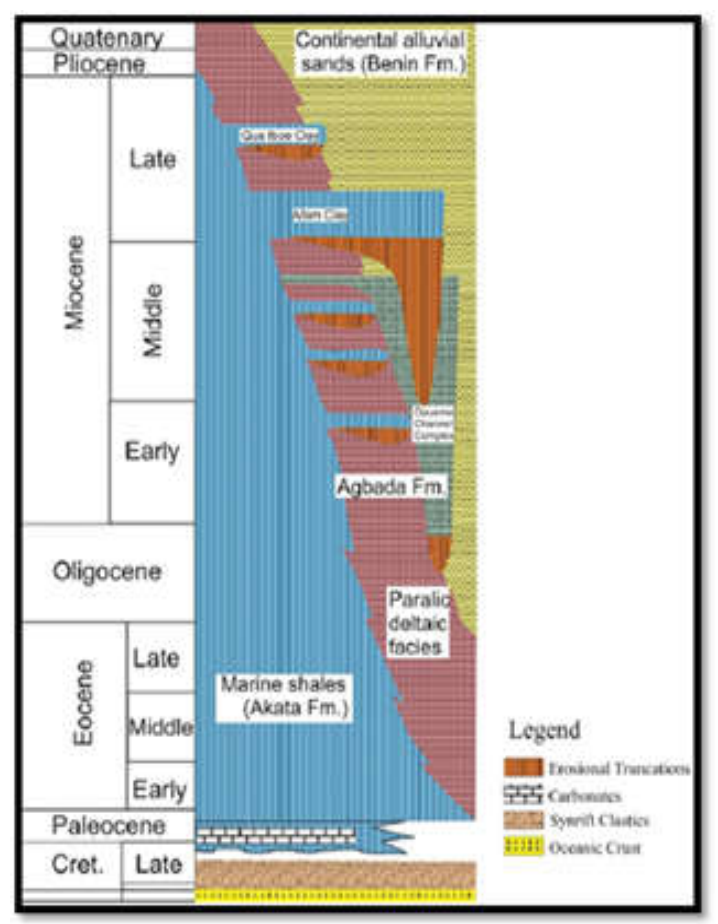

Fig. 2: Schematic representation of the diachronous nature of major lithofacies units, and the stratigraphic relationships of clay-filled channels on the delta flanks. Modified after Doust and Omatsola, (1990) and Lawrence et al., (2002).

Geochemical Results (Major Oxide): Table 2 shows the geochemical composition of analyzed samples from the studied FE-1 well with the average data of Upper Continental Crust (UCC) after McLennan, (2001) and Post-Archean Australian average Shale after Taylor and McLennan, (1985) included. The results of the major oxides shows an enriched $\mathrm{SiO}_{2}$ (81.10 - 90.40) wt. \% with an average of 87.10 wt. \%. Silica enrichment is a measure of sandstone maturity, and is a reflection of the duration and intensity of weathering and destruction of other minerals during transportation. Lindsey, (1999). The value of $\mathrm{Al}_{2} \mathrm{O}_{3}$ content ranges from 2.37 wt. \% to 6.82 wt. \%. As indicated by Cingolani et al., (2003), alteration of rocks during weathering results in depletion of alkalis and alkaline earth elements and preferential enrichment of $\mathrm{Al}_{2} \mathrm{O}_{3}$. The value of $\mathrm{Fe}_{2} \mathrm{O}_{3}$ content ranges from 2.23 wt. $\%$ to 7.78 wt. $\%$ and $\mathrm{TiO}_{2}$ from $0.17-1.36$ wt. \%. Other oxides such as $\mathrm{MgO}(0.11-$ 0.65 wt. \%), $\mathrm{CaO}\left(0.10\right.$ - 0.82 wt. \%), $\mathrm{Na}_{2} \mathrm{O}(0.08$ 0.99 wt. \%) and $\mathrm{K}_{2} \mathrm{O}(0.35-1.72$ wt. \%) are strongly depleted while $\mathrm{MnO}$ and $\mathrm{P}_{2} \mathrm{O}_{5}$ have low concentrations that are less than 1 . The low values might probably be due to chemical destruction under oxidizing condition during weathering. From the foregoing, the average value of silica (87.1 wt. \%) in FE-1 well is far higher 
than the average values of the rest oxides likely due to weathering and diagenetic processes, as sedimentary rocks are enhanced in silica and depleted in iron, magnesium, calcium, sodium, and potassium related to the parent rocks. Also, the average data of the major oxides shows that $\mathrm{SiO}_{2}$ is higher when compared with PASS and UCC values while the rest oxides are lower than the PASS and UCC values.

Trace Elements Result: As specified by Taylor and McLennan, (1985), the behavior of trace elements during sedimentary processes is complex due to factors including weathering, physical sorting, adsorption, provenance, diagenesis and metamorphism. From the result in table 3, the average values of $\mathrm{Ba}$ (Barium) and $\mathrm{Pb}$ (Lead) concentration are enriched when compared with those of PAAS and UCC. The general enrichment in Barium may be due to their deposition in a shallow marine condition. Nagarajan et al., (2007). Among the high field strength elements, $\mathrm{Zr}$ (Zirconium), $\mathrm{Hf}$ (Hafnium) and $\mathrm{Nb}$ (Niobium) concentrations are enriched when compared with PAAS and UCC owing to their link with heavy minerals and are also known to be enhanced in felsic rock than that of mafic rocks. The average values of the transition trace elements (TTE) (Sc, V, Cu, Co and $\mathrm{Zn}$ ) are depleted when compared with PASS and UCC. The general depletion of Scandium is suggesting a relatively felsic source. Cullers and Berendsen, (1998). Transition elements $\mathrm{Cr}$ and $\mathrm{Ni}$ are depleted in respect to PAAS but slightly enriched comparable to UCC.

The depletion of these elements shows a negligible role of the basic rocks as sources of the rocks. Th (Thorium) concentration is also depleted when compared with PASS and UCC. Sr (Strontium) is depleted when related with PAAS and UCC. According to Wronkiewicz and Condie, (1987), weathering studies show that $\mathrm{Sr}$ is rapidly lost during chemical weathering and the amount of this elemental lost is proportional to the degree of weathering.

\begin{tabular}{|c|c|c|c|}
\hline Rarestion & $\begin{array}{c}\text { Deght } \\
\text { (f) }\end{array}$ & Lith dog & LTHOS TRITIGRPHY \\
\hline \multirow{19}{*}{$\frac{2}{2}$} & & 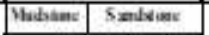 & \multirow{7}{*}{ 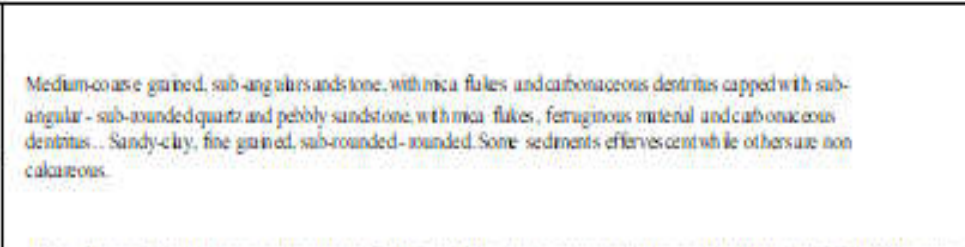 } \\
\hline & & 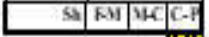 & \\
\hline & 400 & \multirow{12}{*}{ 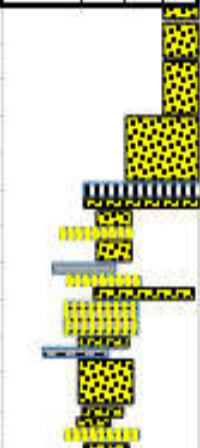 } & \\
\hline & 80 & & \\
\hline & cow & & \\
\hline & wa & & \\
\hline & 200 & & \\
\hline & 240 & & 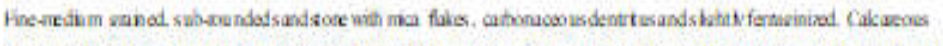 \\
\hline & 20 & & \multirow{2}{*}{ 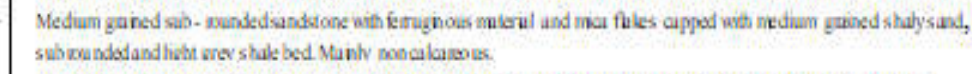 } \\
\hline & 520 & & \\
\hline & wa & & \multirow{4}{*}{ 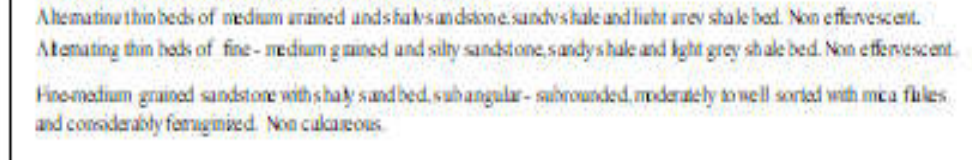 } \\
\hline & 400 & & \\
\hline & 400 & & \\
\hline & 40 & & \\
\hline & $\$ 20$ & [efid & \multirow{2}{*}{ 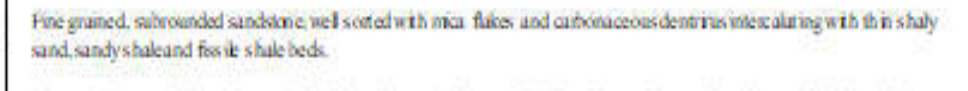 } \\
\hline & 50 & 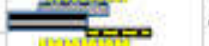 & \\
\hline & $a \times$ & ---3 & \multirow{3}{*}{ 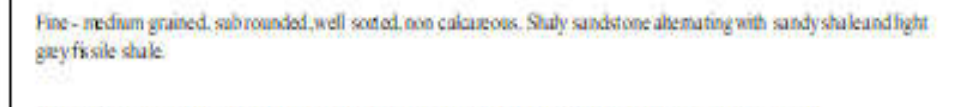 } \\
\hline & -400 & 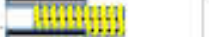 & \\
\hline & $\omega 8$ & thing & \\
\hline \multirow{9}{*}{$\frac{a}{2}$} & 720 & $1---5$ & \multirow[t]{2}{*}{ 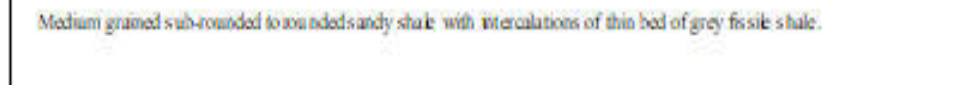 } \\
\hline & 700 & $-7 \mathrm{mon}$ & \\
\hline & 800 & 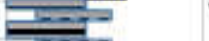 & \multirow{4}{*}{ 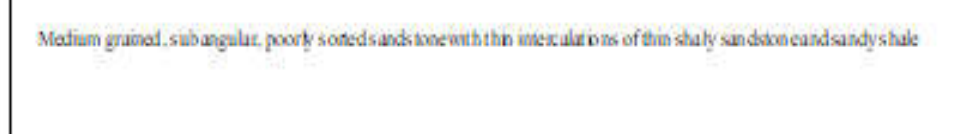 } \\
\hline & sio & Fithitis: & \\
\hline & $\$ \infty$ & $\overline{2}$ & \\
\hline & 90 & 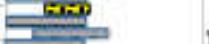 & \\
\hline & 90 & 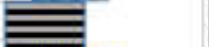 & Gey, fsorit stak \\
\hline & now & & \multirow[t]{2}{*}{ 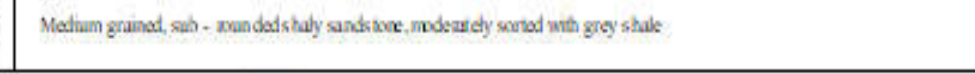 } \\
\hline & $n_{40}$ & & \\
\hline LE & & MLSTONF & $\because=-$ SANDYSHLE WHW SANDSTONE IIIII \\
\hline
\end{tabular}


Table 2: Major oxides geochemical composition of FE-1 Well. UCC - Upper Continental Crust (after Mclenna, 2001) and PAAS - Post Archean Australian Average Shale (after Taylor and Mclenna, 1985).

\begin{tabular}{|c|c|c|c|c|c|c|c|c|c|c|c|c|c|c|c|}
\hline Major oxides (wt. \%) & FE1 & FE2 & FE3 & FE4 & FE5 & FE6 & FE7 & FES & FE9 & FE10 & FE11 & FE12 & Average & PASS & UCC \\
\hline $\mathrm{SiO}_{2}$ & 90.20 & 90.40 & 91.40 & 91.10 & 91.20 & 90.70 & 82.80 & 81.10 & 83.20 & 84.60 & 81.80 & 86.30 & 87.10 & 62.80 & 66.00 \\
\hline $\mathrm{TH}_{2}$ & 0.18 & 0.17 & 0.19 & 0.17 & 0.18 & 0.18 & 0.99 & 0.97 & 0.83 & 1.00 & 1.36 & 1.09 & 0.61 & 0.99 & 0.68 \\
\hline $\mathrm{Al}_{2} \mathrm{O}_{3}$ & 2.48 & 2.37 & 2.59 & 2.56 & 2.58 & 2.59 & 4.81 & 6.82 & 5.81 & 3.40 & 3.32 & 2.52 & 3.49 & 18.90 & 15.20 \\
\hline $\mathrm{Fe}_{2} \mathrm{O}_{3}$ & 2.26 & 2.23 & 3.03 & 3.01 & 3.01 & 3.01 & 5.22 & 6.72 & 4.99 & 5.53 & 7.78 & 4.98 & 4.31 & 7.22 & 5.03 \\
\hline $\mathrm{MnO}$ & 0.03 & 0.02 & 0.03 & 0.03 & 0.03 & 0.02 & 0.05 & 0.05 & 0.05 & 0.05 & 0.04 & 0.04 & 0.00 & 0.11 & 0.08 \\
\hline $\mathrm{MgO}$ & 0.12 & 0.11 & 0.14 & 0.14 & 0.11 & 0.12 & 0.65 & 0.52 & 0.54 & 0.56 & 1.26 & 0.65 & 0.41 & 2.20 & 2.20 \\
\hline $\mathrm{CaO}$ & 0.18 & 0.18 & 0.10 & 0.11 & 0.11 & 0.11 & 0.55 & 0.76 & 0.81 & 0.82 & 1.27 & 0.56 & 0.46 & 1.30 & 4.20 \\
\hline $\mathrm{Na}_{2} \mathrm{O}$ & 0.08 & 0.09 & 0.80 & 0.70 & 0.80 & 0.80 & 0.75 & 0.71 & 0.92 & 0.77 & 0.99 & 0.72 & 0.68 & 1.20 & 3.90 \\
\hline $\mathrm{K}_{2} \mathrm{O}$ & 0.35 & 0.36 & 0.36 & 0.36 & 0.35 & 0.35 & 1.59 & 1.61 & 1.59 & 1.63 & 1.72 & 1.46 & 0.98 & 3.70 & 3.40 \\
\hline $\mathrm{P}_{2} \mathrm{O}_{5}$ & 0.02 & 0.02 & 0.03 & 0.03 & 0.03 & 0.03 & 0.10 & 0.23 & 0.21 & 0.13 & 0.26 & 0.11 & 0.09 & 0.16 & 0.15 \\
\hline
\end{tabular}

Table 3: Trace Elements Composition of FE-1 Well.

\begin{tabular}{|c|c|c|c|c|c|c|c|c|c|c|c|c|c|c|c|}
\hline Trace Element (ppm) & FE1 & FE2 & FE3 & FE4 & FE5 & FE6 & FE7 & FE8 & FE9 & FE10 & FE11 & FE12 & Average & PAAS & $\mathrm{UCC}$ \\
\hline Ag (Silver) & -0.1 & -0.1 & -0.1 & 0.2 & 0.1 & 0.1 & 1 & -0.1 & 1 & 1 & 1 & -0.1 & 0.3 & & \\
\hline As (Arsenic) & 1.1 & 1.2 & 1.3 & 1.3 & 1.2 & 1.4 & 11.6 & 3.8 & 5.2 & 6.7 & 5.7 & 3.9 & 3.7 & & 1.5 \\
\hline $\mathrm{Ba}$ (Barium) & 655 & 680 & 700 & 740 & 750 & 670 & 883 & 1670 & 966 & 820 & 1439 & 1250 & 935.3 & 650 & 550 \\
\hline Be (Beryllium) & 0.5 & 0.6 & 0.7 & 0.8 & 0.8 & 0.7 & 2.8 & 2.2 & 3.2 & 3.6 & 2.6 & 2.5 & 1.8 & & \\
\hline Bi (Bis muth) & 0.03 & 0.04 & 0.02 & 0.04 & 0.05 & 0.03 & 0.24 & 0.14 & 0.18 & 0.16 & 0.17 & 0.23 & 0.1 & & \\
\hline Cd (Cadmium) & -0.1 & -0.1 & 0.1 & 0.1 & 0.1 & \begin{tabular}{|l|}
-0.1 \\
\end{tabular} & 1.1 & -0.1 & 0.1 & 0.1 & 0.2 & -0.1 & 0.1 & & \\
\hline Ce (Cerium) & 111 & 126 & 122 & 90 & 80 & 81 & 142 & 100 & 110 & 129 & 105 & 141 & 111.4 & 79.6 & 64 \\
\hline Co (Cobalt) & 3.6 & 3.6 & 3.5 & 3.5 & 3.5 & 3.6 & 13.5 & 13.5 & 13.2 & 13.4 & 11.1 & 12.9 & 8.2 & 23 & 10 \\
\hline Cr (Chromium) & 20 & 22 & 24 & 21 & 21 & 23 & 104 & 63 & 78 & 92 & 60 & 69 & 49.8 & 110 & 35 \\
\hline Cs (Caesium) & 0.49 & 0.51 & 0.46 & 0.44 & 0.45 & 0.44 & 4.92 & 2.7 & 2.6 & 3.8 & 3.1 & 3.1 & 1.9 & & 3.7 \\
\hline $\mathrm{Cu}$ (Copper) & 10 & 9 & 11 & 12 & 7 & 8 & 22 & 20 & 21 & 22 & 20 & 18 & 15 & 50 & 25 \\
\hline Ga (Gallium) & 4.1 & 4.1 & 4.2 & 4.3 & 4.3 & 4.1 & 28.3 & 18.7 & 19.2 & 18.3 & 26.1 & 27.4 & 13.6 & 17.50 & \\
\hline Hf(Hafnium) & 5.91 & 9.15 & 6.12 & 7.14 & 6.22 & 11.1 & 8.71 & 10.1 & 9.11 & 8.68 & 12.1 & 12.6 & 8.9 & 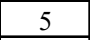 & 5.8 \\
\hline In (Indium) & -0.1 & -0.1 & -0.1 & -0.1 & -0.1 & -0.1 & -0.1 & -0.1 & -0.1 & \begin{tabular}{|l|}
-0.1 \\
\end{tabular} & -0.1 & -0.1 & -0.1 & & \\
\hline La (Lanthanum) & 10.2 & 9.8 & 9.5 & 11.4 & 10.9 & 12.1 & 70.0 & 49.9 & 55.2 & 58.7 & 63.2 & 69.1 & 35.8 & 38.2 & 30 \\
\hline Mo (Molybdenum) & 4.1 & 3.2 & 3.8 & 5.1 & 5.3 & 3.7 & 3 & 4.5 & 4.1 & 5.6 & 7.1 & 7.3 & 4.7 & & \\
\hline $\mathrm{Nb}$ (Niobium) & 5.22 & 5.84 & 6.11 & 6.34 & 6.73 & 5.12 & 41 & 33.9 & 39.3 & 38.1 & 50.2 & 60.1 & 24.8 & 1.9 & 25 \\
\hline \begin{tabular}{|l}
$\mathrm{Ni}$ (Nickel) \\
\end{tabular} & 16 & 15 & 14 & 17 & 16 & 17 & 34 & 24 & 21 & 28 & 23 & 21 & 20.5 & 55 & 20 \\
\hline $\mathrm{Pb}$ (Lead) & 22 & 28 & 31 & 36 & 29 & 41 & 32 & 38 & 33 & 30 & 37 & 29 & 32.2 & 20 & \\
\hline Sb (Antimony) & 0.1 & 0.1 & 0.1 & 0.2 & 0.2 & 0.2 & 0.6 & 0.1 & 0.2 & 0.4 & 0.3 & 0.6 & 0.3 & & 0.2 \\
\hline Sc (Scandium) & 2 & 2 & 2 & 2 & 2 & 2 & 14 & 9 & 11 & 10 & 13 & 14 & 6.9 & 16 & 11 \\
\hline Sn (Tin) & 1.1 & 1.2 & 1.1 & 1 & 1 & 1.1 & 4.4 & 3.4 & 3.6 & 4.2 & 3.8 & 3.6 & 2.5 & & \\
\hline Sr (Stro & 38.2 & 39.2 & 40.1 & 40.8 & 41.3 & 37.1 & 142 & 177 & 182 & 199 & 119 & 290 & 112.1 & 200 & 350 \\
\hline $\mathrm{Ta}(\mathrm{Ta}$ & 0.46 & 0.48 & 0.51 & 0.53 & 0.44 & 0.45 & 2.73 & 2.2 & 3.8 & 4 & 2.6 & 2.8 & 1.8 & & 2.2 \\
\hline $\mathrm{Te}$ (Tellurium) & -0.2 & -0.2 & -0.2 & -0.2 & -0.2 & -0.2 & -0.2 & -0.2 & -0.2 & -0.2 & -0.2 & -0.2 & -0.2 & & \\
\hline Th (Thorium) & 3.67 & 3.74 & 3.39 & 3.63 & 3.22 & 3.58 & 18.50 & 13.70 & 14.20 & 16.10 & 14.30 & 15.20 & 9.40 & 14.6 & 10.7 \\
\hline Ti (Titanium) & 1080 & 1110 & 1118 & 1120 & 1030 & 1090 & 8100 & 6590 & 5987 & 7342 & 8000 & 87200 & 3547.3 & & \\
\hline $\mathrm{Tl}(\mathrm{Th}$ & -0.2 & -0.2 & -0.2 & -0.2 & -0.2 & \begin{tabular}{|l|}
-0.2 \\
\end{tabular} & 0.6 & 0.4 & 0.6 & 0.6 & 0.4 & 0.4 & 0.2 & & \\
\hline U (Uranium) & 0.85 & 0.91 & 0.99 & 0.92 & 0.89 & 1 & 3.90 & 3.20 & 3.32 & 3.76 & 3.45 & 3.80 & 2.20 & 3.1 & 2.8 \\
\hline $\mathrm{V}$ (Vanadium) & 18.3 & 19.2 & 20.1 & 22.4 & 21.6 & 17.8 & 127 & 81.2 & 77.3 & 78.9 & 100.2 & 110.2 & 57.9 & 150 & 60 \\
\hline W (Tungsten) & 1 & 1 & 1 & 1 & 1 & 1 & 2.5 & 3.5 & 3 & 2.8 & 2.6 & 2.5 & 1.9 & & \\
\hline $\mathrm{Y}$ (Yttrium) & 5.19 & 6.2 & 6.3 & 6.1 & 6.4 & 5.11 & 33.9 & 27.9 & 29.2 & \begin{tabular}{|l|}
28.4 \\
\end{tabular} & 30.8 & 31.3 & 18.1 & 27 & 22 \\
\hline Zn (Zinc) & 18 & 21 & 20 & 24 & 16 & 19 & 120 & 135 & 86 & 98 & 128 & 130 & 67.9 & 85 & 71 \\
\hline Zr (Zirconium) & 250 & 284 & 310 & 370 & 440 & 430 & 320 & 404 & 380 & 390 & 520 & 401 & 374.9 & 210 & 190 \\
\hline
\end{tabular}


Table 4: Some trace element ratios and weathering indices of the studied FE-1 well samples. Source: Table 2 and 3.

\begin{tabular}{|c|c|c|c|c|c|c|c|c|c|c|c|c|c|c|c|}
\hline Ratios & FE1 & FE2 & FE3 & FE4 & FE5 & FE6 & FE7 & \begin{tabular}{|l|l|} 
FE8 \\
\end{tabular} & FE9 & \begin{tabular}{|l|} 
FE10 \\
\end{tabular} & FE11 & FE12 & Average & PASS & UCC \\
\hline $\log \left(\mathrm{SiO}_{2} / \mathrm{Al}_{2} \mathrm{O}_{3}\right)$ & 1.56 & 1.58 & 1.55 & 1.55 & 1.55 & 1.54 & 1.24 & 1.08 & 1.16 & 1.40 & 1.40 & 1.53 & 1.43 & 0.52 & 0.64 \\
\hline $\log \left(\mathrm{K}_{2} \mathrm{O} / \mathrm{Na}_{2} \mathrm{O}\right)$ & 0.64 & 0.60 & -0.35 & -0.29 & -0.36 & -0.36 & 0.33 & 0.36 & 0.24 & 0.33 & 0.24 & 0.31 & 0.14 & 0.49 & -0.06 \\
\hline $\log \left(\mathrm{Fe}_{2} \mathrm{O}_{3} / \mathrm{K}_{2} \mathrm{O}\right)$ & 0.81 & 0.79 & 0.93 & 0.93 & 0.93 & 0.93 & 0.52 & 0.62 & 0.50 & 0.54 & 0.66 & \begin{tabular}{|l|}
0.53 \\
\end{tabular} & 0.72 & 0.29 & 0.17 \\
\hline $\log \left(\mathrm{Na}_{2} \mathrm{O} / \mathrm{K}_{2} \mathrm{O}\right)$ & -0.64 & $\begin{array}{ll}-0.60 \\
\end{array}$ & 0.35 & 0.29 & 0.36 & 0.36 & -0.33 & -0.36 & -0.24 & \begin{tabular}{|l|}
-0.33 \\
\end{tabular} & -0.24 & -0.31 & -0.14 & -0.49 & 0.06 \\
\hline $\mathrm{Al}_{2} \mathrm{O}_{3}+\mathrm{Na}_{2} \mathrm{O}+\mathrm{K}_{2} \mathrm{O}$ & 2.91 & 2.82 & 3.75 & 3.62 & 3.73 & 3.74 & 7.15 & 9.14 & 8.32 & 5.80 & 6.03 & 4.70 & 5.14 & 23.80 & 22.50 \\
\hline $\mathrm{K}_{2} \mathrm{O} / \mathrm{Na}_{2} \mathrm{O}$ & 4.38 & 4.00 & 0.80 & 0.51 & 0.44 & 0.44 & 2.12 & \begin{tabular}{|l|}
2.27 \\
\end{tabular} & 1.73 & \begin{tabular}{|l|l|}
2.12 \\
\end{tabular} & 1.74 & \begin{tabular}{|l|}
2.03 \\
\end{tabular} & 1.88 & 3.08 & 0.87 \\
\hline $\mathrm{Th} / \mathrm{Cr}$ & 0.18 & 0.17 & 0.14 & \begin{tabular}{l|l}
0.17 \\
\end{tabular} & 0.15 & 0.16 & 0.18 & \begin{tabular}{|l|}
0.22 \\
\end{tabular} & 0.18 & \begin{tabular}{|l|l|}
0.18 \\
\end{tabular} & \begin{tabular}{|l|}
0.24 \\
\end{tabular} & \begin{tabular}{|l|l|}
0.22 \\
\end{tabular} & 0.18 & \begin{tabular}{|l|}
0.13 \\
\end{tabular} & 0.31 \\
\hline $\mathrm{La} / \mathrm{Sc}$ & 5.10 & \begin{tabular}{l|l}
4.90 \\
\end{tabular} & 4.75 & \begin{tabular}{l|l|}
5.70 \\
\end{tabular} & 5.45 & 6.05 & 5.00 & \begin{tabular}{|l|}
5.54 \\
\end{tabular} & 5.02 & \begin{tabular}{|l|}
5.87 \\
\end{tabular} & \begin{tabular}{|l|}
4.86 \\
\end{tabular} & \begin{tabular}{|l|}
4.94 \\
\end{tabular} & 5.23 & \begin{tabular}{|l|}
2.39 \\
\end{tabular} & 2.73 \\
\hline $\mathrm{Th} / \mathrm{Sc}$ & 1.84 & 1.87 & 1.70 & \begin{tabular}{l|l}
1.82 \\
\end{tabular} & 1.61 & 1.79 & 1.32 & \begin{tabular}{|l|}
1.52 \\
\end{tabular} & 1.29 & \begin{tabular}{|l|}
1.61 \\
\end{tabular} & \begin{tabular}{|l|}
1.10 \\
\end{tabular} & \begin{tabular}{|l|}
1.09 \\
\end{tabular} & 1.55 & \begin{tabular}{|l|}
0.91 \\
\end{tabular} & 0.97 \\
\hline $\mathrm{Th} / \mathrm{Co}$ & 1.02 & 1.04 & 0.97 & 1.04 & 0.92 & 0.99 & 1.37 & \begin{tabular}{|l|}
1.01 \\
\end{tabular} & 1.08 & \begin{tabular}{|l|}
1.20 \\
\end{tabular} & \begin{tabular}{|l|}
1.29 \\
\end{tabular} & \begin{tabular}{|l|l|}
1.18 \\
\end{tabular} & 1.09 & \begin{tabular}{|l|}
0.63 \\
\end{tabular} & 1.07 \\
\hline $\mathrm{Cr} / \mathrm{Th}$ & 5.45 & 5.88 & \begin{tabular}{l|l|}
7.08 \\
\end{tabular} & 5.79 & 6.52 & 6.42 & 5.62 & \begin{tabular}{|l|}
4.60 \\
\end{tabular} & 5.50 & |5.71 & 4.20 & |4.54 & 5.61 & \begin{tabular}{|l|}
7.53 \\
\end{tabular} & 3.27 \\
\hline $\mathrm{U} / \mathrm{Th}$ & 0.23 & 0.24 & 0.29 & 0.25 & 0.28 & 0.28 & 0.21 & \begin{tabular}{|l|}
0.23 \\
\end{tabular} & 0.23 & \begin{tabular}{|l|}
0.23 \\
\end{tabular} & \begin{tabular}{|l|}
0.24 \\
\end{tabular} & \begin{tabular}{|l|}
0.25 \\
\end{tabular} & 0.25 & 0.21 & 0.26 \\
\hline $\mathrm{Ni} / \mathrm{Co}$ & 4.40 & 4.17 & 4.00 & \begin{tabular}{l|l|}
4.85 \\
\end{tabular} & 4.57 & 4.72 & 2.52 & \begin{tabular}{|l|}
1.78 \\
\end{tabular} & 1.60 & \begin{tabular}{|l|}
2.09 \\
\end{tabular} & \begin{tabular}{|l|}
2.07 \\
\end{tabular} & \begin{tabular}{|l|l|}
1.63 \\
\end{tabular} & 3.20 & \begin{tabular}{|l|}
2.39 \\
\end{tabular} & 2.00 \\
\hline $\mathrm{Cu} / \mathrm{Zn}$ & 0.56 & 0.43 & 0.55 & 0.50 & 0.44 & 0.42 & 0.18 & \begin{tabular}{|l|}
0.15 \\
\end{tabular} & 0.24 & \begin{tabular}{|l|}
0.22 \\
\end{tabular} & \begin{tabular}{|l|}
0.16 \\
\end{tabular} & \begin{tabular}{|l|}
0.14 \\
\end{tabular} & 0.33 & \begin{tabular}{|l|}
0.56 \\
\end{tabular} & 0.35 \\
\hline $\mathrm{V} / \mathrm{Cr}$ & 0.92 & 0.87 & 0.84 & 1.07 & 1.03 & 0.77 & 1.22 & \begin{tabular}{|l|}
1.23 \\
\end{tabular} & 0.99 & \begin{tabular}{|l|l|}
0.86 \\
\end{tabular} & 1.67 & \begin{tabular}{|l|}
1.59 \\
\end{tabular} & 1.09 & $\begin{array}{ll}1.36 \\
\end{array}$ & 1.71 \\
\hline $\mathrm{V} /(\mathrm{V}+\mathrm{Ni})$ & 0.53 & 0.56 & 0.58 & 0.06 & 0.50 & 0.51 & 0.79 & \begin{tabular}{|l|}
0.77 \\
\end{tabular} & 0.07 & \begin{tabular}{|l|}
0.74 \\
\end{tabular} & \begin{tabular}{|l|}
0.81 \\
\end{tabular} & \begin{tabular}{|l|}
0.84 \\
\end{tabular} & 0.56 & \begin{tabular}{|l|}
0.73 \\
\end{tabular} & 0.75 \\
\hline $\mathrm{Al}_{2} \mathrm{O}_{3} / \mathrm{SiO}_{2}$ & 0.03 & 0.03 & 0.03 & 0.03 & 0.03 & 0.03 & 0.06 & 0.08 & 0.07 & \begin{tabular}{|l|}
0.04 \\
\end{tabular} & 0.04 & \begin{tabular}{|l|}
0.03 \\
\end{tabular} & 0.04 & 0.30 & 0.23 \\
\hline $\mathrm{Fe}_{2} \mathrm{O}_{3}+\mathrm{MgO}$ & 2.38 & 2.34 & 3.17 & 3.15 & 3.12 & 3.13 & 5.87 & 7.24 & 5.53 & 6.09 & 9.04 & 5.63 & 4.72 & 9.42 & 7.23 \\
\hline $\mathrm{SiO}_{2} / \mathrm{Al}_{2} \mathrm{O}_{3}$ & 36.37 & 38.14 & 35.29 & 35.59 & 35.35 & 35.02 & 17.21 & 11.89 & 14.32 & 24.88 & 24.64 & 34.24 & 28.58 & 3.32 & 4.34 \\
\hline $\mathrm{Fe}_{2} \mathrm{O}_{3} / \mathrm{K}_{2} \mathrm{O}$ & 6.46 & 6.19 & 8.42 & 8.36 & 8.60 & 8.60 & 3.28 & \begin{tabular}{|l|l|}
4.17 \\
\end{tabular} & 3.14 & \begin{tabular}{|l|}
3.39 \\
\end{tabular} & \begin{tabular}{|l|}
4.52 \\
\end{tabular} & \begin{tabular}{|l|}
3.41 \\
\end{tabular} & 5.71 & 1.95 & 1.48 \\
\hline $\mathrm{Na}_{2} \mathrm{O}+\mathrm{K}_{2} \mathrm{O}$ & 0.43 & 0.45 & 1.16 & 1.06 & 1.15 & 1.15 & 2.34 & 2.32 & 2.51 & \begin{tabular}{|l|}
2.40 \\
\end{tabular} & 2.71 & \begin{tabular}{|l|}
2.18 \\
\end{tabular} & 1.66 & 4.90 & 7.30 \\
\hline $\mathrm{Al}_{2} \mathrm{O}_{3} /\left(\mathrm{CaO}+\mathrm{MgO}+\mathrm{Na}_{2} \mathrm{O}+\mathrm{K}_{2} \mathrm{O}\right)$ & 3.39 & 3.20 & 1.85 & 1.95 & 1.88 & 1.87 & 1.36 & 1.89 & 1.51 & 0.89 & 0.63 & 0.74 & 1.76 & 2.25 & 1.11 \\
\hline$\left(\mathrm{Fe}_{2} \mathrm{O}_{3}+\mathrm{MgO}\right) /\left(\mathrm{K}_{2} \mathrm{O}+\mathrm{Na}_{2} \mathrm{O}\right)$ & 5.53 & 5.20 & 2.73 & 2.97 & 2.71 & 2.72 & 2.51 & 3.12 & 2.20 & \begin{tabular}{|l|l}
2.54 \\
\end{tabular} & 3.34 & 2.58 & 2.84 & 1.92 & 0.99 \\
\hline
\end{tabular}

Provenance: The geochemical characteristics of a rock could be used to determine the provenance of a rock. Both major oxides and trace elements composition of rock have been extensively used to describe the provenance nature. Chen et al., (2016); Mitra, (2018). $\mathrm{Cr}, \mathrm{Ni}$ are utmost significant source rock indicator owing to their low movement during sedimentary processes.

McLennan et al., (1990). Armstrong-Altrin et al., (2004), submitted that when $\mathrm{Cr}>150 \mathrm{ppm}$ and $\mathrm{Ni}>100$ ppm in concentration, it infers mafic or ultramafic provenance.

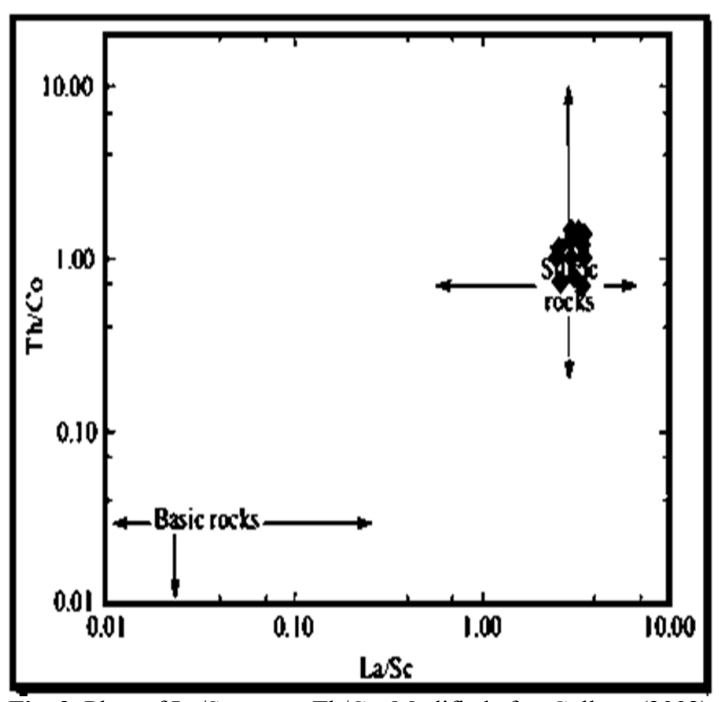

Fig. 3. Plots of La/Sc versus Th/Co. Modified after Cullers, (2002).

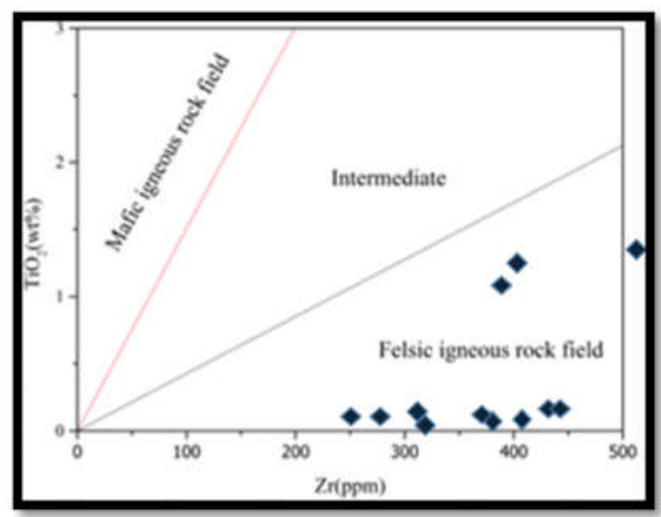

Fig. 4. Plots of $\mathrm{Zr}$ versus $\mathrm{TiO}_{2}$. Modified after Hayashi et al., (1997).

However, the average value of $\mathrm{Cr}(49.80 \mathrm{ppm})$ and $\mathrm{Ni}$ $(20.50 \mathrm{ppm})$ in table 3 have low concentrations which confirm a felsic source area and not mafic or ultramafic rocks as the source areas. Bivariate plot of $\mathrm{La} / \mathrm{Sc}$ versus Th/Co, Cullers, (2002), (Fig. 3) and $\mathrm{Zr}$ versus $\mathrm{TiO}_{2}$, Hayashi et al., (1997), (Fig. 4), establishes that the source of FE-1 well sediment are from a felsic rock. Table 5 denotes a series of trace element ratios (Th/Sc, Th/Co, Th/Cr, Cr/Th, La/Sc) of the FE-1 well sediment in association with trace elemental ratios of the clastics derivative from felsic and mafic rocks which indicates a discrete separation in their values. Cullers, (2000). Evaluation of these elemental ratios with the FE-1 well sediments' elemental ratio evidently reveals that the FE-1 well sediment are derived from felsic rock. 
Table 5: Range of elemental ratios of FE-1 well sediment compared to the ratios derived from felsic and mafic rocks (Cullers, 2000), Upper Continental Crust (UCC) and Post-Archean Australian Average Shale (PAAS) Taylor and McLennan, (1985).

\begin{tabular}{|c|c|c|c|c|c|}
\hline \multirow{2}{*}{ Elemental Ratio } & Ranges of value in FE-1 well & \multicolumn{2}{|c|}{ Range of Sediments } & Upper Continental \\
\cline { 2 - 4 } & $(\mathrm{n}=12)$ & Felsic rock & Mafic rock & $\begin{array}{c}\text { Post-Archean Australian } \\
\text { Crust (UCC) }\end{array}$ & 0.9 \\
\hline $\mathrm{Th} / \mathrm{Sc}$ & $1.09-1.87$ & $0.84-20.5$ & $0.05-0.22$ & 0.79 & 0.63 \\
\hline $\mathrm{Th} / \mathrm{Co}$ & $0.92-1.37$ & $0.67-19.4$ & $0.04-1.4$ & 0.63 & 0.13 \\
\hline $\mathrm{Th} / \mathrm{Cr}$ & $0.14-0.23$ & $0.13-2.7$ & $0.018-0.046$ & 0.13 & 7.53 \\
\hline $\mathrm{Cr} / \mathrm{Th}$ & $4.20-7.08$ & $4.0-15$ & $25-100$ & 7.76 & 2.4 \\
\hline $\mathrm{La} / \mathrm{Sc}$ & $4.75-6.05$ & $2.5-16.3$ & $0.43-0.86$ & 2.21 & Shale (PAAS) \\
\hline \multicolumn{7}{|c|}{$\mathrm{n}=$ number of samples } \\
\hline
\end{tabular}

Chemical classification: The concentrations of these major oxide groups (silica and alumina, alkali oxides, and iron oxide plus magnesia) have been used to classify sandstones. E.g Herron, (1988) and Lindsey, (1999). The plot of $\log \left(\mathrm{SiO}_{2} / \mathrm{Al}_{2} \mathrm{O}_{3}\right)$ versus $\log$ $\left(\mathrm{Fe}_{2} \mathrm{O}_{3} / \mathrm{K}_{2} \mathrm{O}\right)$ diagram by Herron, (1988) classifies the FE-1 well samples mainly as quartzarenites with some Fe-rich sands. Figure 5.

From the interpretation of the reference set proposed by Lindsey, (1999), the succeeding rules for the chemical classification of sandstones are:

1. $\log \left(\mathrm{SiO}_{2} / \mathrm{Al}_{2} \mathrm{O}_{3}\right) \geq 1.5$, then the sandstone is classified as Quartzarenite

2. Greywacke, $\log \left(\mathrm{SiO}_{2} / \mathrm{Al}_{2} \mathrm{O}_{3}\right)<1$ and $\log$ $\left(\mathrm{K}_{2} \mathrm{O} / \mathrm{Na}_{2} \mathrm{O}\right)<0$

3. $\log \left(\mathrm{SiO}_{2} / \mathrm{Al}_{2} \mathrm{O}_{3}\right)<1.5, \log \left(\mathrm{K}_{2} \mathrm{O} / \mathrm{Na}_{2} \mathrm{O}\right) \geq 0$ and $\log$ $\left(\left(\mathrm{Fe}_{2} \mathrm{O}_{3}+\mathrm{MgO}\right) /\left(\mathrm{K}_{2} \mathrm{O}+\mathrm{Na}_{2} \mathrm{O}\right)\right)<0$, Arkose (including subarkose)

4. $\log \left(\mathrm{SiO}_{2} / \mathrm{Al}_{2} \mathrm{O}_{3}\right)<1.5$ and either $\log \left(\mathrm{K}_{2} \mathrm{O} / \mathrm{Na}_{2} \mathrm{O}\right)<$ 0 or $\log \left(\left(\mathrm{Fe}_{2} \mathrm{O}_{3}+\mathrm{MgO}\right) /\left(\mathrm{K}_{2} \mathrm{O}+\mathrm{Na}_{2} \mathrm{O}\right)\right) \geq 0$. Lithic arenite (Sub greywacke).

Established on the above sets, the average value of $\log$ $\left(\mathrm{SiO}_{2} / \mathrm{Al}_{2} \mathrm{O}_{3}\right)$ for the FF-1 sediment is 1.43 which is $<1.5$. Consequently, set 1 is ruled out. Condition 2 is also ruled out because the average value of $\log$ $\left(\mathrm{K}_{2} \mathrm{O} / \mathrm{Na}_{2} \mathrm{O}\right)$ for the FE-1 sediment is 0.14 , which is $>$ 0 . In the reference set 3 , the average values obtained for $\log \left(\mathrm{SiO}_{2} / \mathrm{Al}_{2} \mathrm{O}_{3}\right)$ is 1.43 and $\log \left(\mathrm{K}_{2} \mathrm{O} / \mathrm{Na}_{2} \mathrm{O}\right)$ is 0.14 whereas the average value of $\log \left(\left(\mathrm{Fe}_{2} \mathrm{O}_{3}+\right.\right.$ $\left.\mathrm{MgO}) /\left(\mathrm{K}_{2} \mathrm{O}+\mathrm{Na}_{2} \mathrm{O}\right)\right)$ is 2.84 . These values obtained for FE-1 sediments cannot be characterized as Arkose. The mean values achieved correspond with $\log$ $\left(\mathrm{SiO}_{2} / \mathrm{Al}_{2} \mathrm{O}_{3}\right)<1.5$ and $<\quad 10 \mathrm{~g}$ $\left(\left(\mathrm{Fe}_{2} \mathrm{O}_{3}+\mathrm{MgO}\right) /\left(\mathrm{K}_{2} \mathrm{O}+\mathrm{Na}_{2} \mathrm{O}\right)\right)>0$, this falls in the fourth set. Therefore, it submit lithic arenites for the sediments under study. The general quartz content in lithic arenites is from $30-80 \%$ while the lithic fragments is from $5-50 \%$. The nature of the lithic fragments determines the compositional maturity and the textures varies from sub-mature to mature. Quartzarenite are found to contain more than $95 \%$ silica and less than $15(\%)$ matrix material and are formed along continental margin, where there is no active tectonic activity thereby favouring large weathering, long abrasion, sorting and multiple sequences of weathering and transportation.

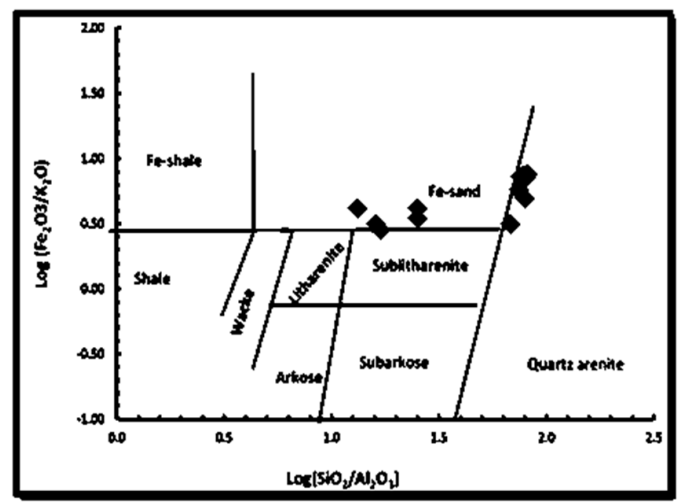

Fig 5. Chemical classification of the FE-1 sediment based on log $\left(\mathrm{SiO}_{2} / \mathrm{Al}_{2} \mathrm{O}_{3}\right)$ versus $\log \left(\mathrm{Fe}_{2} \mathrm{O}_{3} / \mathrm{K}_{2} \mathrm{O}\right)$ diagram of Herron, (1988).

Tectonic Setting: The chemical compositions of clastic rocks are significantly controlled by plate tectonic settings of their provenances, consequently clastic rocks from different tectonic settings possess terrainspecific geochemical signatures. Roser and Korsch, (1986). As proposed by Maynard et al., (1982), the $\log \left(\mathrm{K}_{2} \mathrm{O} / \mathrm{Na}_{2} \mathrm{O}\right)$ ratio versus $\mathrm{Log}\left(\mathrm{SiO}_{2} / \mathrm{Al}_{2} \mathrm{O}_{3}\right)$ ratio is used to determine the tectonic setting of clastic terrigenous sedimentary rocks. The recognized tectonic settings on the discrimination diagram of Maynard et al., (1982) are: A1 - arc setting and andesitic detritus; A2 - evolved arc setting, felsic pluton detritus; ACM - Active continental margin; PM - passive margin. When utilized for the samples of FE1 well, they plotted mainly in the passive margin zone which infer that the tectonic setting for FE-1 well facies is in the passive continental margin. Fig. 6. In addition, the plot of Roser and Korsch, (1986), was also utilized to determine the tectonic setting of FE-1 well sediments. The recognized tectonic settings on the $\mathrm{K}_{2} \mathrm{O} / \mathrm{Na}_{2} \mathrm{O}$ versus $\mathrm{SiO}_{2}$ discrimination diagram of Roser and Korsch, (1986) are: the passive margin $(\mathrm{PM})$, active continental margin (ACM) and oceanic island arc (OIA). When utilized for the samples under study, they plotted mainly in the passive margin zone 
which also infer that the tectonic setting for FE-1 well facies is passive margin. Fig. 7.

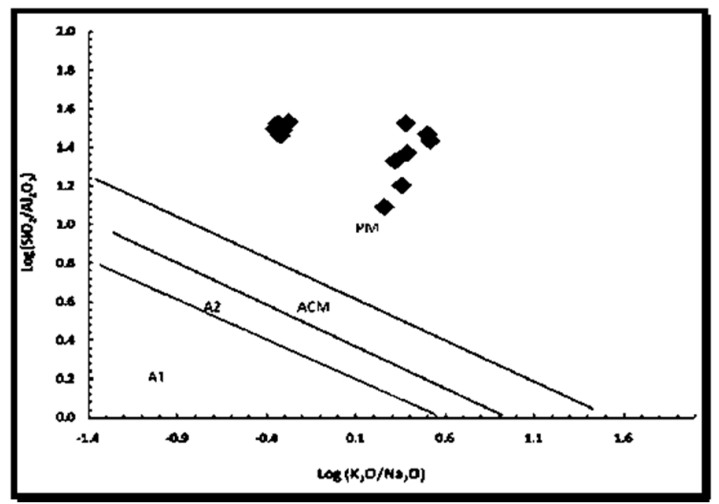

Fig 6: $\mathrm{Log}\left(\mathrm{K}_{2} \mathrm{O} / \mathrm{Na}_{2} \mathrm{O}\right)$ versus $\log \left(\mathrm{SiO}_{2} / \mathrm{Al}_{2} \mathrm{O}_{3}\right)$ tectonic discrimination diagram of FE-1 well. After Maynard et al., (1982). $\mathrm{Al}$ - arc setting, basaltic and andesitic detritus, A2 - evolved arc setting, felsic pluton detritus, ACM - Active continental margin, PM - Passive Continental Margin.

Paleo oxygen Condition: The trace elements data (Table 3) and numerous geochemical ratios generally used to disclose the paleo-redox conditions during sedimentation of siliciclastic rocks are shown in table 4.

Authors such as Jones and Manning (1994) and Nath et al., (1997), have shown that trace element ratios such as $\mathrm{Ni} / \mathrm{Co}, \mathrm{V} / \mathrm{Cr}, \mathrm{V} /(\mathrm{V}+\mathrm{Ni})$ and $\mathrm{U} / \mathrm{Th}$ can be used to appraise paleo oxygen conditions of ancient sediment. Trace elements like U, Th, $\mathrm{Cu}, \mathrm{Zn}, \mathrm{Ni}, \mathrm{Co}$, $\mathrm{V}$ and $\mathrm{Cr}$ present an important role in understanding the paleo redox condition of sediment. Sediment deposited in oxidizing environment generally has a low content of $U$, while those deposited in oxygen minimum zone (OMZ) reveal higher content of $U$. Armstrong- Altrin et al., (2015a). Consequently, the average low $U$ content (average $2.25 \mathrm{ppm}$ ) of the FE1 well sediments submit an oxygenated condition during deposition.

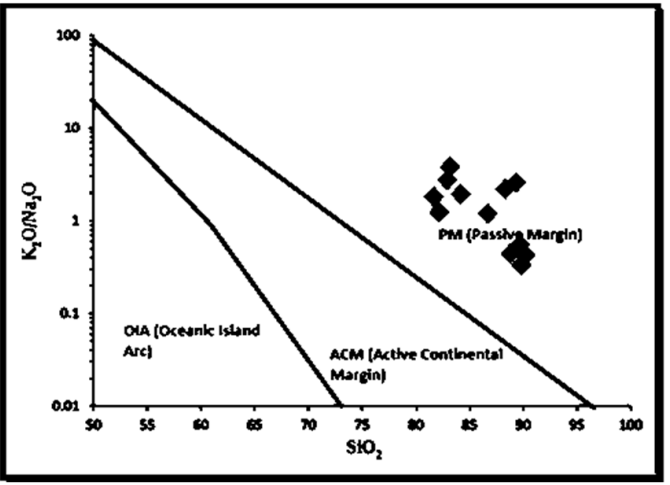

Fig 7: Plot of $\mathrm{K}_{2} \mathrm{O} / \mathrm{Na}_{2} \mathrm{O}$ versus $\mathrm{SiO}_{2}$ tectonic discrimination for FE-1 well sediment. Modified after Roser and Korsch, (1986).
Given to Nath et al., (1997), the ratio of uranium to thorium could be used as a redox indicator. Where the $\mathrm{U} / \mathrm{Th}$ ratios is below 0.75 , it infers oxic conditions of deposition, while values from $0.75-1.25$ indicates dyoxic, values greater than 1.25 infers suboxic to anoxic conditions. Applying this to the studied sediments, the calculated U/Th ratios of FE-1 well shows low values from $0.2-0.3$ with an average value of 0.2 which infer that the sediments under study were deposited in oxic environments of deposition. Cobalt (Co) is a valuable indicator for depositional conditions and is usually enriched in comparison with $\mathrm{Ni}$ in oxic conditions.

Jones and Manning, (1994). Co concentrations in all the analyzed samples are relatively low and range from 3.50 to $13.50 \mathrm{ppm}$. (Table 3). According to Jones and Manning, (1994), when the ratio of $\mathrm{Ni} / \mathrm{Co}$ is below 5, it indicates oxic environments, whereas ratios from 5 7, it suggest dyoxic, ratios greater than 7 infers suboxic to anoxic environments. The ratio of $\mathrm{Ni} / \mathrm{Co}$ in $\mathrm{FE}-1$ well ranges from 1.6 - 4.9 with an average value of 3.2 which indicate oxic environments of deposition. Chromium (Cr) is understood to be related only with the detrital faction and is not directly influenced by redox conditions. Author such as Nagarajan et al., (2007) have used $\mathrm{V} / \mathrm{Cr}$ ratio as an indicator of paleo oxygen condition of sediment deposition. Agreeing to Jones and Manning, (1994), when the value of $\mathrm{V} / \mathrm{Cr}$ ratio is below 2, it denote oxic conditions while value above 2 denote anoxic conditions.

The $\mathrm{V} / \mathrm{Cr}$ ratios in FE-1 sediments range from 0.8 to 1.7 , with an average value of 1.1 which indicates that the sediments were deposited in an oxic depositional condition. Additionally, the $\mathrm{Cu} / \mathrm{Zn}$ ratio is also used as a redox constraint. High $\mathrm{Cu} / \mathrm{Zn}$ ratios infer reducing depositional conditions whereas low $\mathrm{Cu} / \mathrm{Zn}$ ratios propose oxidizing conditions. Deng and Qian, (1993). $\mathrm{Cu} / \mathrm{Zn}$ ratio in this study varies from 0.1 to 0.6 with an average of 0.3 which indicates that the samples of FE1 well were deposited under oxidizing environments.

As specified by Suttner and Dutta, (1986), the binary plot of $\mathrm{Ni} / \mathrm{Co}$ versus $\mathrm{V} / \mathrm{Cr}$ can indicate redox condition. When applied to FE-1 well sediment, it indicates oxic condition during deposition. Fig. 8. However, the binary plot of $\mathrm{Ni} / \mathrm{Co}$ versus $\mathrm{V} /(\mathrm{V}+\mathrm{Ni})$ by Rimmer, (2004), indicates oxic to anoxic condition during deposition of FE-1 well sediment. Fig. 9. The cross plot of vanadium versus nickel of FE-1 samples from the studied area shows that the sediments had mixed marine and terrigenous source input and were deposited under oxic to dyoxic condition. Kafousia, et al., (2010). Fig. 10. 


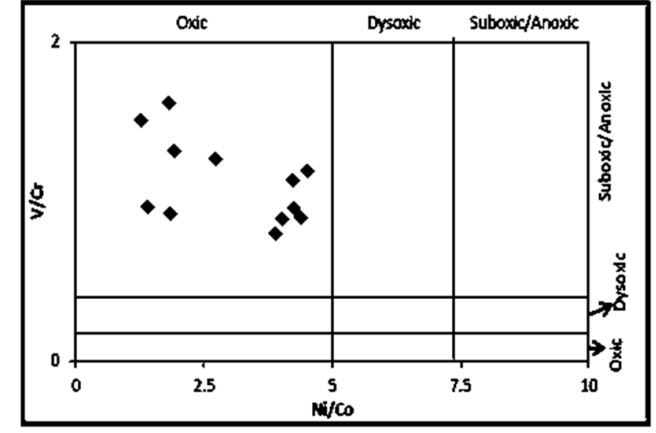

Fig.8: Binary plot of $\mathrm{Ni} / \mathrm{Co}$ versus $\mathrm{V} / \mathrm{Cr}$ indicates oxic condition during deposition of FE-1 well sediment. Modified after Suttner and Dutta (1986).

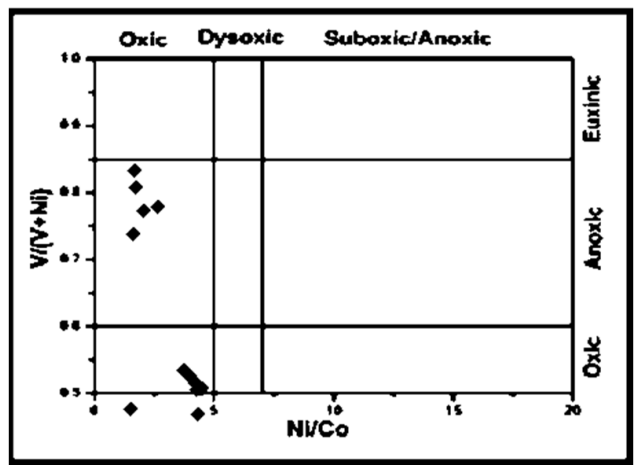

Fig. 9: Binary plot of $\mathrm{Ni} / \mathrm{Co}$ versus $\mathrm{V} /(\mathrm{V}+\mathrm{Ni})$ indicates oxic to anoxic condition during deposition of FE-1 well sediment. Modified after Rimmer, (2004).

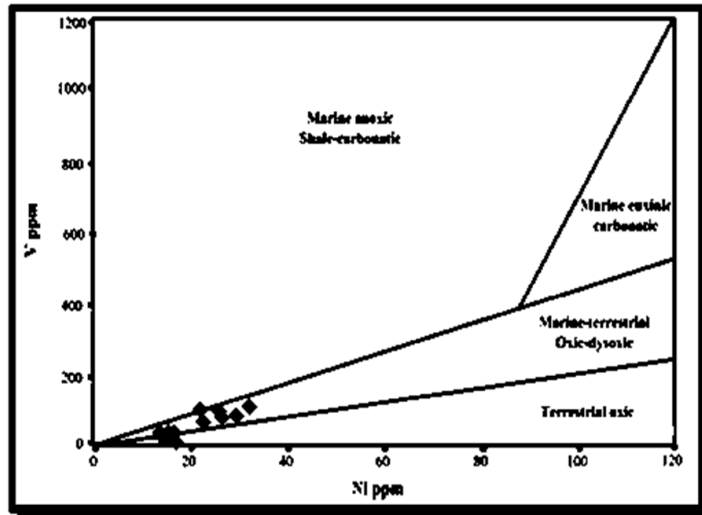

Fig. 10: The Cross plot of vanadium versus nickel of FE-1 samples from studied area shows that the sediments had mixed marine and terrigenous source input and were deposited under oxic to dyoxic condition. Modified after Kafousia, et al., (2010).

Maturity and Palaeoclimate during Sedimentation: The plot of $\mathrm{SiO}_{2}$ versus $\mathrm{Al}_{2} \mathrm{O}_{3}+\mathrm{K}_{2} \mathrm{O}+\mathrm{Na}_{2} \mathrm{O}$ in Fig. 11 illustrates that majority of the sediments are formed under humid/semi-humid climatic zone. This wet condition quickens weathering process and increase the speed of chemical maturity. As posited by Potter,
(1978), $\mathrm{SiO}_{2} / \mathrm{Al}_{2} \mathrm{O}_{3}$ index is used to show the extent of sandstones maturity in relation to mineralogical maturity. Maturity is revealed best in quartz, rock fragments, feldspars and grain size.

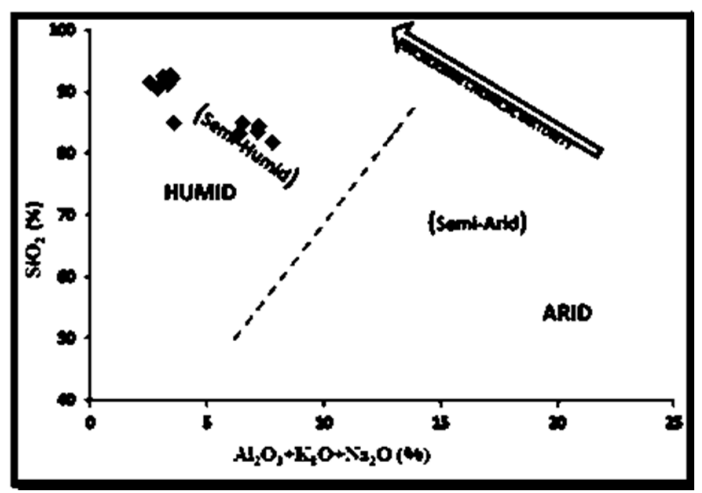

Fig. 11: Binary plot of $\mathrm{SiO}_{2}$ versus $\left(\mathrm{Al}_{2} \mathrm{O}_{3}+\mathrm{Na}_{2} \mathrm{O}+\mathrm{K}_{2} \mathrm{O}\right)$ indicates deposition mainly in humid climate. Modified after Suttner and Dutta (1986).

Increasing sediment maturity can be determine by the quartz contents in the sediments, because quartz survives differently to feldspars, mafic minerals and lithics. Roser et al., (1996). The average $\mathrm{SiO}_{2} / \mathrm{Al}_{2} \mathrm{O}_{3}$ ratios in an intact igneous rocks range from $\sim 3.0$ (basic rocks) to $\sim 5.0$ (acidic rocks). When the values of $\mathrm{SiO}_{2} / \mathrm{Al}_{2} \mathrm{O}_{3}$ ratio is $>5.0$ in samples, it suggests progressive maturity. Roser et al., (1996). High ratios designate mineralogically mature (quartzose, rounded) samples, while low ratios characterizes chemically immature samples. Potter, (1978). The $\mathrm{SiO}_{2} / \mathrm{Al}_{2} \mathrm{O}_{3}$ ratio for the FE-1 well samples have ranges of 11.89 38.14 with an average of 28.58 . The values is $>5.0$ which indicates that the samples are mineralogically mature. The high values obtained from the ratio of $\mathrm{SiO}_{2} / \mathrm{Al}_{2} \mathrm{O}_{3}$ shows that all the samples have low degree of clayness. The higher the $\mathrm{SiO}_{2}$ content, the lower the degree of clayness. $\mathrm{Na}_{2} \mathrm{O}+\mathrm{K}_{2} \mathrm{O}$ (alkali content) shows the degree of the feldspar content and also pertinent for index of chemical maturity. The $\mathrm{Na}_{2} \mathrm{O}+\mathrm{K}_{2} \mathrm{O}$ content varies between 0.43 and 2.71, with an average of 1.66 . This indicates the occurrence of feldspars and low chemical maturity. The $\mathrm{Fe}_{2} \mathrm{O}_{3} / \mathrm{K}_{2} \mathrm{O}$ ratio varies from 3.14 to 8.60 with an average of 5.71. The samples studied have a much higher $\mathrm{SiO}_{2} / \mathrm{Al}_{2} \mathrm{O}_{3}$ ratio and low $\mathrm{Fe}_{2} \mathrm{O}_{3} / \mathrm{K}_{2} \mathrm{O}$ ratio, thus, they are mineralogically more stable and less prone to reactivity during supercritical $\mathrm{CO}_{2}$ exposure. Farquhar et al., (2014). From the ratios values, the sediment under examination are therefore mineralogically mature.

The $\mathrm{Al}_{2} \mathrm{O}_{3} /\left(\mathrm{CaO}+\mathrm{MgO}+\mathrm{Na}_{2} \mathrm{O}+\mathrm{K}_{2} \mathrm{O}\right)$ ratio can be used in determining the stability of mobile oxides as proposed by Gill and Yemane, (1996). The positive 
values obtained are in the range of $0.63-3.39$, which deduce that there are stable mobile oxides in the FE-1 well samples.

Conclusion: Sedimentological analysis revealed five main lithofacies. Major oxides and trace elemental ratios clearly reveals the mixed marine and terrigenous source input sediment are derived from felsic rock in passive margin zone deposited mainly in humid/semihumid climatic condition. Also, the trace elemental ratios of $\mathrm{U} / \mathrm{Th}, \mathrm{Ni} / \mathrm{Co}, \mathrm{Cu} / \mathrm{Zn}, \mathrm{V} / \mathrm{Cr}$ and binary plot of $\mathrm{Ni} / \mathrm{Co}$ versus $\mathrm{V} / \mathrm{Cr}$ indicated an oxic environment of deposition. However, the binary plot of $\mathrm{Ni} / \mathrm{Co}$ versus $\mathrm{V} /(\mathrm{V}+\mathrm{Ni})$ indicates oxic to anoxic condition during deposition. The samples studied are mineralogically mature with stable mobile oxides.

Acknowledgments: The authors sincerely acknowledge the Nigerian Petroleum Development Company (NPDC) of Nigeria for the provision of the ditch cutting samples for this research work. Our cardinal thanks are due Professor Odedede of Delta State University, for his advice and guidance during the research work.

\section{REFERENCES}

Adenugba, AA; Dipo, SO (2013). Non-oil exports in the economic growth of Nigeria: a study of agricultural and mineral resources. J Educ Soc Res 3: $403-418$.

Adebayo, OF (2011). Biostratigraphy, Sequence Stratigraphic Analysis of Three Offshore Wells from the Niger Delta, Nigeria. Ph.D, University of Ado Ekiti, Ado Ekiti, Nigeria.

Armstrong-Altrin, JS; Lee, YL; Verma, SP; Ramasamy, S (2004). Geochemistry of sandstones from the upper Miocene Kudankulam formation, Southern India: Implications for provenance, weathering and tectonic setting. Jour. Sediment Res., 74: 285 - 297.

Avbovbo, AA; Ayoola, EO (1981). Petroleum prospects of southern Nigeria's Anambra Basin. Oil and Gas. 334 - 347

Cingolani, CA; Manassero, M; Abre, P (2003). Composition, provenance, and tectonic setting of Ordovician siliciclastic rocks in the San Rafael block: southern extension of the Precordillera crustal fragment, Argentina: J. of South Ameri. Earth Sci. 16(1): 91 - 106.

Cullers, RL (2002). Implications of elemental concentrations for provenance, redox conditions, and metamorphic studies of shales and limestones near Pueblo, CO, USA: Chemical Geology, 191(4): 305 - 327.

Deng, HW; Qian, K (1993). Analysis on sedimentary geochemistry and environment", Science Technology Press, Gansu, 15 - 85.

Gill, S; Yemane, K (1996). Implications of a Lower Pennsylvanian Ultisol for equatorial pangean climates and early oligotrophic forest ecosystems. Geology, 24 (10): 905 - 908

Hayashi, K; Fujisawa, H; Holland, H; Ohmoto, H (1997). Geochemistry of 1.9 Ga sedimentary rocks from Northeastern Labrador, Canada: Geochimica st Cosmochimica Acta. 61 (19): 4115 $-4137$.

Herron, MM (1988). Geochemical classification of terrigenous sands and shales from core or log data. Journal of Sedimentary petrology. 58(5): 820 829.

Hosper, J (1965). Gravity field and structure of the Niger Delta, Nigeria, West Africa. Geol Soc Am Bull 76: 407 - 422

Jones, B; Manning, DC (1994). Comparison of geochemical indices used for the interpretation of paleo-redox conditions in Ancient mudstones: Chemical Geology, 111(1-4): 111 - 129.

Kafousia, N; Karakitios, V; Jenkyns, HC (2010). Preliminary data from the first record of the Toarcian Oceanic Anoxic Event in the sediments of the Pindos Zone (Greece). Bulletin of the Geol. society of Greece, 43(2): 627 - 633

Kaplan, A; Lusser, CU; Norton, IO (1994). Tectonic Map of the World, Panel 10. Scale 1:10,000,000. Tulsa, OK, USA: Ameri. Asso. of Pet. Geologists.

Knox, GJ; Omatsola, EM (1987). Development of the Cenozoic Niger Delta in terms of the Escalator Regression model and impact on hydrocarbon distribution, proceedings of the KNGMG Symposium: Coastal Lowlands, Geology and Geotechnology, Kluwer Academic Publishers, The Netherlands, 181 - 202.

Kulke, H (1995). Nigeria. In: Kulke H, editor. Regional Petroleum Geology of the World. Part II: Africa, America, Australia and Antarctica. Berlin, Germany: Gebruder Borntraeger, 143 172.

Lindsey, DA (1999). An Evaluation of Alternative Chemical Classifications of Sandstones. United State Geological Survey Open-File Report. 99 346. 
Maynard, JB; Valloni, R; Yu, HS (1982). Composition of modern deep-sea sands from arc-related basins: in Leggett, J.K., eds., Trench forearc geology: sedimentation and tectonics on modern and ancient active plate margins. Geol. Soc. Lond. Spec. Pub., 10: 551 - 561.

McLennan, SM; Taylor, SR; Mcculloch, MT; Maynard, JB (1990). Geochemical and Nd-Sr isotopic composition of deep sea turbidites: Crustal evolution and plate tectonics associations. Geochimica et Cosmochimica Acta, 54: 2015 2050 .

Mitra, R; Chakrabart, G; Shome, D (2018). Geochemistry of the Palaeo-Mesoproterozoic Tadpatri shales, Cuddapah basin, India: implications on provenance, paleo weathering and paleo redox conditions. Acta Geochim 37(5): 715 -733 .

Nagarajan, R; Madhavaraju, J; Nagendral, R; Armstrong-Altrin, JS; Moutte, J (2007). Geochemistry of Neoproterozoic shales of the Rabanpalli Formation, Bhima Basin, Northern Karnataka, southern India: implications for provenance and paleo redox conditions. Rev Mex Cienc Geol 24: 150 - 160.

Nath, BN; Bau, M; Ramalingeswara, RB; Rao, CM (1997). Trace and rare earth elemental variation in Arabian Sea sediments through a transect across the oxygen minimum zone: Geochimica et Cosmochimica Acta, 61(12): 2375 - 2388.
Nwajide, CS; Reijers, TJA (1996). Sequence architecture in outcrop: examples from the Anambra Basin, Nigeria. NAPE Bulletin 11: 23 32.

Obasi, RA; Madukwe, HY; Olaosun, T (2019). Source area weathering, paleo-environment and paleo climatic conditions of soils from Bitumen Rich Ode Irele Area of Ondo State, Nigeria. European Journal of Engineering Research and Science 4(3): $59-67$.

Reijers, TJA; Petters, SW; Nwajide, CS (1997). The Niger Delta Basin, In: Selley RC, editor, African Basins-Sedimentary Basin of the World 3: Amsterdam, Elsevier Science, pp. 151-172.

Rimmer, SM (2004). Geochemical Paleo redox Indicators in Devonian-Mississippian Black Shales, Central Appalachian Basin (USA). Chemical Geology 206: 373 - 391.

Roser, BP; Korsch, RJ (1986). Determination of tectonic setting of sandstone-mudstone suites using $\mathrm{SiO}_{2}$ content and $\mathrm{K}_{2} \mathrm{O} / \mathrm{Na}_{2} \mathrm{O}$ ratio: Journal of Geology, 94(5): 635 - 650.

Short, KC; Stauble, AJ (1967). Outline of geology of Niger Delta: American Association of Petroleum Geologists Bulletin, 51: 761 - 779.

Suttner, LJ; Dutta, PK (1986). Alluvial sandstones composition and paleo climate, I. Framework mineralogy. J Sediment Res 56(3): 329 - 345.

Whiteman, AJ (1982). Nigeria: It's Petroleum Geology, Resources and Potentials, I \& II, Graham and Trotman, London, 1 - 394. 